\title{
A CMOS Microsystem Combining Magnetic ACtuation And In-Situ Optical Detection of MiCROPARTICLES
}

\author{
U. Lehmann $^{1)}$, M. Sergio ${ }^{2)}$, S. Pietrocola ${ }^{1)}$, C. Niclass ${ }^{2)}$, E. Charbon ${ }^{2)}$ and M.A.M. Gijs ${ }^{1)}$ \\ ${ }^{1}$ Institute of Microelectronics and Microsystems, Microsystems Laboratory 2 \\ ${ }^{2}$ School of Computer and Communication Sciences, \\ Ecole Polytechnique Fédérale de Lausanne (EPFL) \\ Lausanne, SWITZERLAND
}

\begin{abstract}
We present a CMOS-based hybrid microfluidic system that combines the manipulation of magnetic microparticles through a magnetic field with in situ optical detection via single photon avalanche diodes (SPADs). Magnetic particles are actuated within a glass micro-capillary and are detected upon passage over the SPAD, where they block incident light and thus lower the photon count. The optical sensors, which are not influenced by the magnetic actuation forces, allow detecting the presence of magnetic particles of 30,5 and $1 \mu \mathrm{m}$ diameter, while being able to distinguish between different sizes.
\end{abstract}

Keywords: Magnetic Actuation, Optical Detection, CMOS, Single Photon Avalanche Diode

\section{INTRODUCTION}

Magnetic microparticles and their application in microfluidics and bioanalytical microsystems have been steadily gaining interest in recent years [1-3]. The manipulation of such particles can be achieved by modulating the topology of a magnetic field over the chip surface via an array of micro-coils $[4,5]$. This method allows a very precise control of the microparticles and does not require external moving parts. In our system the magnetically actuated particles are detected optically. Thus, advantageously, the principles used for actuation and detection are not coupled and permit high sensitivity. In addition, due to the speed and accuracy of sensing, it is conceivable to implement stable and effective feedback mechanisms to ensure actuation reliability and precision.

\subsection{System Setup}

The CMOS chip presented here comprises four metal layers. The upper two levels form overlapping square coils, while the lower pair contains the SPADs, which are positioned under the opening at the center of each coil. Fig. 1 presents schematic views of the system, while Fig. 2 shows a photomicrograph of the chip with closeups of a coil and a center SPAD.

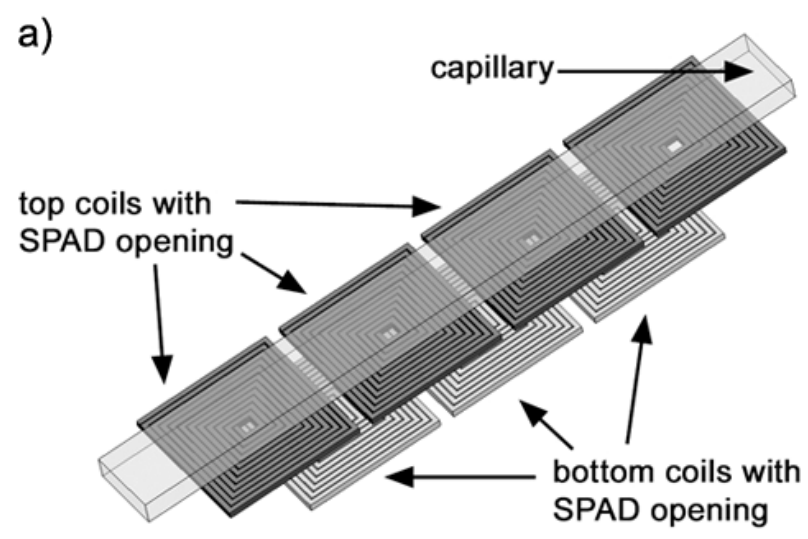

b)

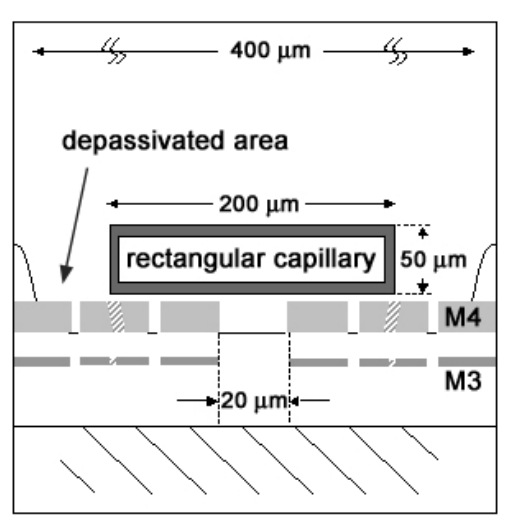

Figure 1: Schematic views of the system. a: Explosion view of the system, where the integrated microcoils generate the field for magnetic actuation and the SPADs at the coil centers perform the optical detection. b) Side view of the chip with micro-capillary. 
The coils are fabricated in $0.35 \mu \mathrm{m} 2 \mathrm{P} 4 \mathrm{M}$ CMOS technology and the SPAD implementation is based on a design similar to [6]. A glass microcapillary (Vitrotube), which is closed at both ends and placed on top of the coils, contains the magnetic particles suspended in an aqueous solution.

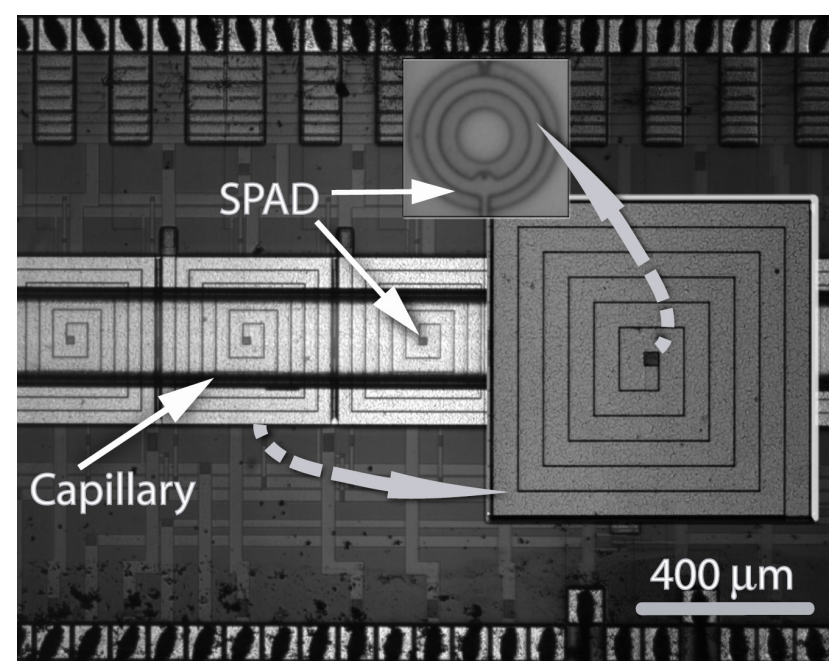

Figure 2. Photograph of the system with glass capillary. The insets show one coil and a SPAD in detail.

The actuation of the magnetic particles is achieved via a three-phase current scheme [5]. If one coil is set to attractive mode, the adjacent coils are both in repulsive mode. To achieve attractive and repulsive modes, coil currents are stirred in opposite directions. The absolute value of the DC current in an active coil is $30 \mathrm{~mA}$. The particles are additionally magnetized via a perpendicular homogeneous magnetic field applied from outside the chip.

\subsection{Single Photon Detection}

For the optical detection we are making use of the advantageous properties of Single Photon Avalanche Diodes (SPADs). A SPAD is a p-n junction, which is reverse biased above breakdown by the by a voltage known as excess bias. This configuration, schematically shown in Fig. 3, causes the optical gain to become virtually infinite, thus making single photon detection possible.

When a photon is absorbed in the multiplication region, an avalanche is triggered. Thanks to a resistance placed in series to the p-n junction, the reverse bias temporarily drops below breakdown and quenches the avalanche.
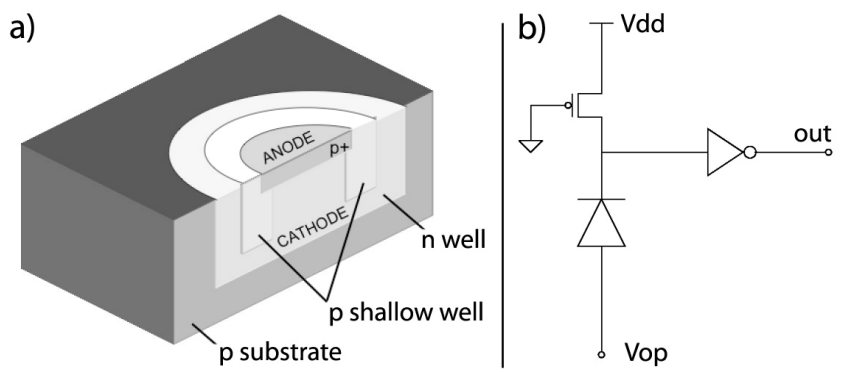

Figure 3. Schematics of a passively quenched $S P A D$. a) $3 D$ view of the CMOS structure, b) schematic diagram.

In this design, avalanche quenching, which in combination with the recharge time determines the dead time and thus the detection cycle of a SPAD, is achieved through a passive method. The measured dead time of $40 \mathrm{~ns}$ resulting in a detection saturation of $25 \mathrm{MHz}$ is well below the time a particle requires for its transit between coil centers. The voltage pulse generated during a detection cycle is regenerated and converted to a digital pulse by an inverter.

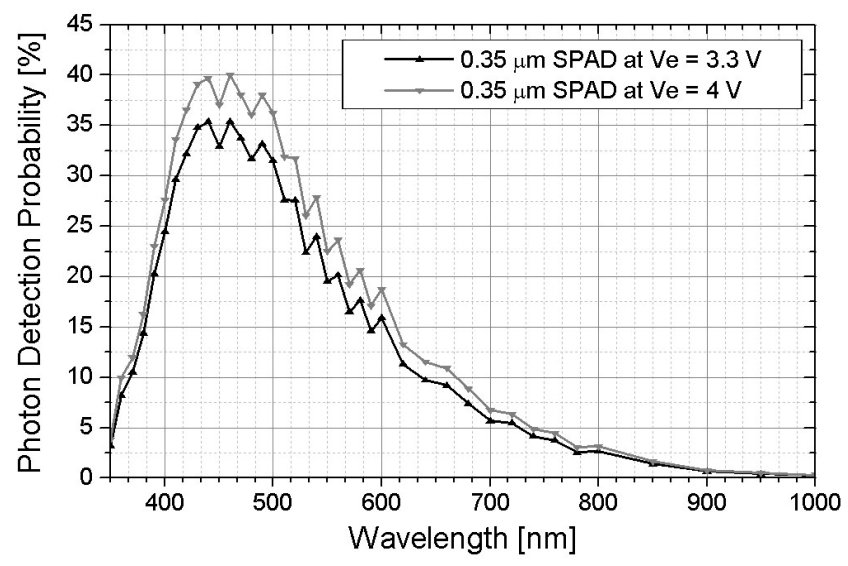

Figure 4. Photon detection probability for different excess bias voltages as a function of the wavelength of impinging photons.

The probability that a digital pulse is generated upon impinging photons, the photon detection probability (PDP), is plotted in Fig. 4 as a function of excess bias voltage and wavelength. The noise caused by a SPAD determines the minimum detectable photon flux and it is characterized by the frequency of spurious pulses. It is known as dark count, its frequency as dark count rate 
(DCR). In this design, it is in average $750 \mathrm{~Hz}$ for an excess bias voltage of $3.3 \mathrm{~V}$ at room temperature.

The detection scheme, depicted in Fig. 5, is based on the idea of the particles casting a shadow on the SPAD when passing over the center of the attracting coil. Thus the number of impinging photons is reduced and the number of counts decreased. The reduction of the photon count can also give an indication towards the particle size.

a)

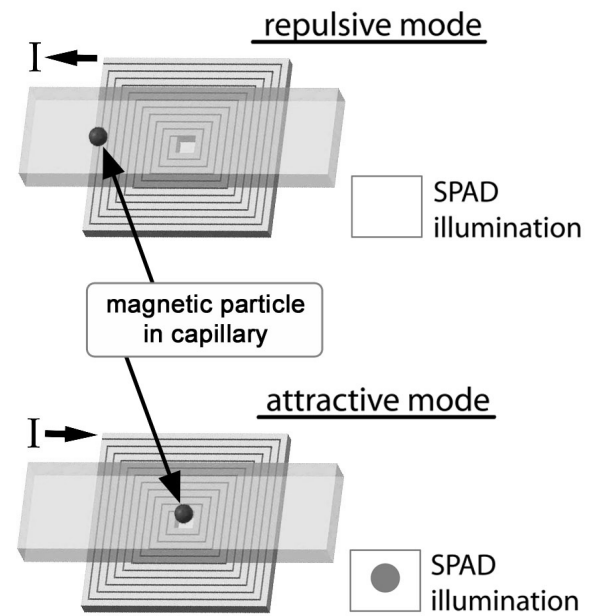

Figure 5. Schematics of the optical detection. A passing particle shields the SPAD from incident light, thus lowering photon count.

\section{EXPERIMENTS AND RESULTS}

We examined the magnetic performance of the system by manipulating magnetic particles of different diameters $(1,5$, and $30 \mu \mathrm{m})$ within a glass micro-capillary. First experiments showed that a certain distance between the coils and the manipulated particles is necessary in order to prevent the particles from being negatively influenced or even trapped by the gap between windings. The walls of the glass capillary have a thickness of 25 up to $100 \mu \mathrm{m}$, which allows smoothening the magnetic field acting on the particles. Thus the magnetic particles can easily follow the field gradient to the next corresponding coil center, where they can be optically detected by a SPAD. All experiments were performed using a standard microscope with incident illumination.

We adjusted the light intensity of the microscope to prevent the optical detectors from going into saturation. All experiments were performed using dispersed magnetic particles, which formed loose cluster over the attractive coils' centers. Fig. 6 presents the particle transport and a plot of the signal obtained from a selected detector during the repeated passage of a group of $30 \mu \mathrm{m}$ magnetic microparticles. It can be seen in the graph that the photon count is significantly lowered while a particle is present over the coil center. This is due to the occlusion of light caused by the particles, as is well in accordance with our expectations. In addition we see that the particle size can influence the strength of the obtained signal. The particles of situation 2 and 4 in Fig. 6 have different sizes and the photon count for the larger particle (2) is smaller than for the particle in situation 4 .

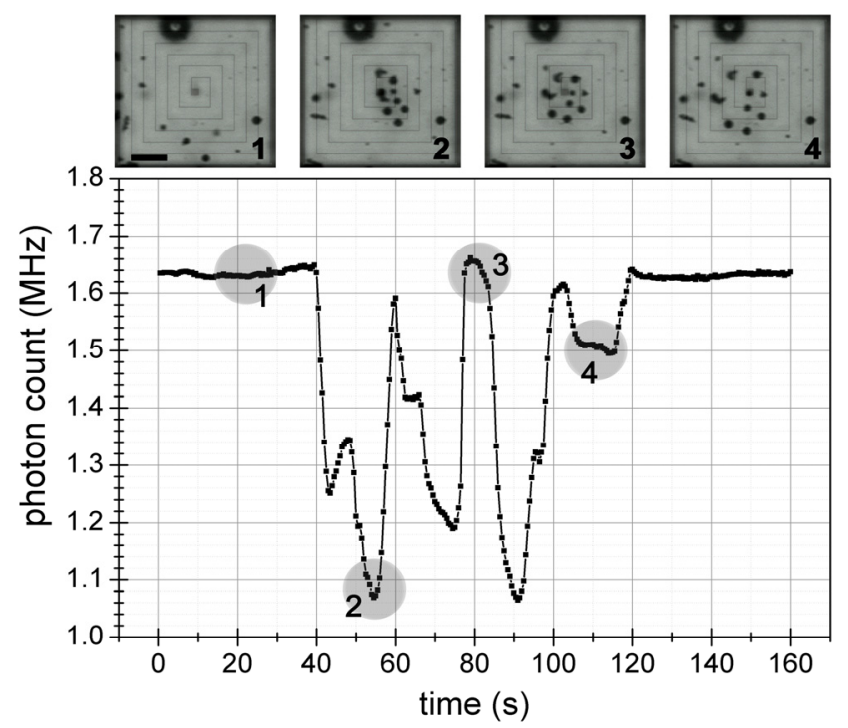

Figure 6. The signal of a single SPAD during the repeated passage of a cloud of particles ( $\varnothing$ ca. 30 $\mu \mathrm{m})$. The photon count varies depending on the position and size of the magnetic particles. The upper part shows photographs of the coil where the signal was recorded. The photographs were taken during the measurement. (Dimension bar: $100 \mu \mathrm{m})$

We examined this effect further using particles of $1 \mu \mathrm{m}$ and $5 \mu \mathrm{m}$ diameter. Fig. 7 shows the signals obtained from these experiments. We normalized the photon count since the starting count differs between experiments. As already observed in earlier experiments, we can see that larger particles show a stronger effect on the 
frequency of events counted by the detector. Hence, the time of occlusion and the number of particles involved can be determined in a strictly quantitative fashion.

We also observed a dependence of the signal on the position of larger particles over the SPAD. Since the active surface of the detector has a diameter of $8 \mu \mathrm{m}$, the signal will vary depending on the fraction of the particle's shadow that falls onto the sensitive surface. This effect is visible in Fig. 7 for the particles of $5 \mu \mathrm{m}$ diameter. Here the curve shows fluctuations of the signal while the particles group over the SPAD. For smaller particles the variation is less important since the shadow is sufficiently small. A comparison with the optical observation indicated that the negative peaks are evoked by single particles. This demonstrates that we are capable of detecting single magnetic particles of a certain minimum size.

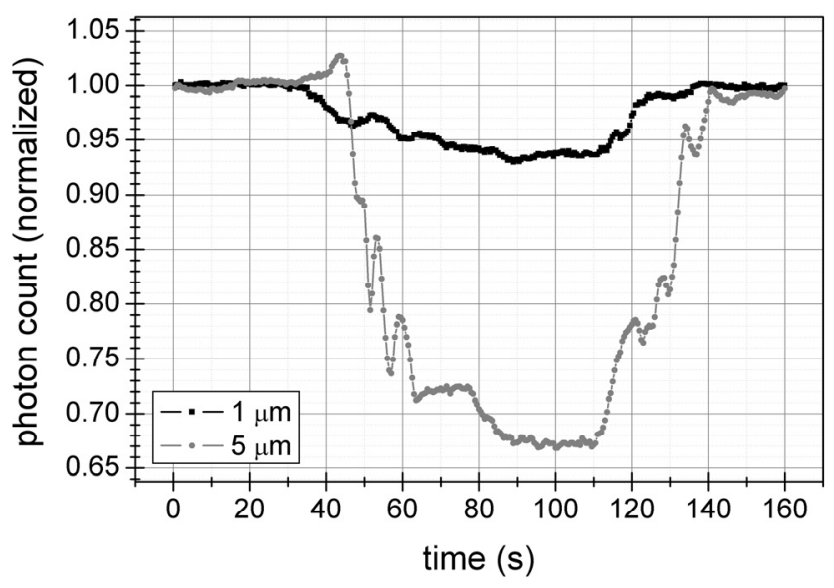

Figure 7. The signal of a SPAD output for different particle sizes. In both cases a cloud of particles was passing over the SPAD, casting shadows of different size and density.

In addition we expect an asymptotic behavior of the particle size sensitivity, since every shadow that is larger than the sensing surface will yield the same signal. This hypothesis needs to be confirmed in future experiments.

\section{CONCLUSION}

Using a CMOS microsystem for magnetic particle actuation with integrated optical detection elements we are able to move and detect magnetic particles of 1 to $30 \mu \mathrm{m}$ diameter. Since the principles of actuation and detection are not coupled, it will be possible in the near future to use the particles as mobile substrates to transport bio-materials of interest, e.g. cells, and to perform on-chip analysis without interference between actuation and detection. In conclusion we can state that the combination of magnetic actuation and integrated optical detection represents a promising approach to miniaturized systems for on-chip bio-analysis.

\section{ACKNOWLEDGEMENTS}

The authors are grateful to Martin Lanz and Giovanni Nicoletti for their technical support. The research was partially supported by a grant of the Swiss National Science Foundation.

\section{REFERENCES}

[1] M. A. M. Gijs, "Magnetic bead handling onchip: new opportunities for analytical applications," Microfluidics and Nanofluidics, vol. 1, pp. 22-40, 2004.

[2] T. Deng, G. M. Whitesides, and M. Radhakrishnan, "Manipulation of magnetic microbeads in suspension using micromagnetic systems fabricated with soft lithography," Appl. Phys. Lett, vol. 78, pp. 1775-1777, 2001.

[3] R. Wirix-Speetjens, W. Fyen, K. D. Xu, J. De Boeck, and G. Borghs, "A force study of onchip magnetic particle transport based on tapered conductors," IEEE Trans. Magn., vol. 41, pp. 4128-4133, 2005.

[4] H. Lee, Y. Liu, R. M. Westervelt, and D. Ham, "IC/Microfluidic hybrid system for magnetic manipulation of biological cells," IEEE Journal of Solid-State Circuits, vol. 41, pp. 1471-1480, 2006.

[5] A. Rida, V. Fernandez, and M. A. M. Gijs, "Long-range transport of magnetic microbeads using simple planar coils placed in a uniform magnetostatic field," Appl. Phys. Lett., vol. 83, pp. 2396, 2003.

[6] C. Niclass, M. Sergio, and E. Charbon, "A single photon avalanche diode array fabricated in $0.35-\mu \mathrm{m}$ CMOS and based on an eventdriven readout for TCSPC experiments", Proc. SPIE 6372, 63720S, 2006. 